Poincare Journal of Analysis E Applications

Vol. 2015 (2), Special Issue (IWWFA-II, Delhi), 65-75

(C) Poincare Publishers

\title{
O-FRAMES FOR OPERATORS IN BANACH SPACES
}

\author{
Oleg Reinov \\ Date of Receiving : $\quad$ 31.03.2015 \\ Date of Acceptance : 10.10 .2015
}

\begin{abstract}
A new notion of $\mathrm{O}$-frame for an operator $T: X \rightarrow Y$ in Banach spaces is introduced: we say that a system $\mathcal{F}:=\left(\left(x_{k}^{\prime}\right)_{k=1}^{\infty},\left(y_{k}\right)_{k=1}^{\infty}\right)$ is $\mathrm{O}$-frame (operator frame) for $T$, if for every $x \in X$ the series $\sum_{k=1}^{\infty}\left\langle x_{k}^{\prime}, x\right\rangle y_{k}$ converges in $Y$ and

$$
T x=\sum_{k=1}^{\infty}\left\langle x_{k}^{\prime}, x\right\rangle y_{k}, x \in X .
$$

This is an evident, but, as we hope, interesting generalization of the well known notion of a Banach (Schauder) frame. We used the classical tools as well as (and mainly - also now classical) the technique, developed by Aleksander Pełczyński, to get first general properties of O-frames.

We present a small survey on the topic. Let us mention only that, in particular, as in the case of the identity operators, it can be proved that

(i) an operator has an operator frame if and only if

(ii) it factors through a Banach space with a basis, and, when it is defined on a separable Banach space, iff

(iii) it has the bounded approximation property.

Also, we consider a new notion of an unconditional operator frame and present some similar results.
\end{abstract}

\section{O-frames}

We use the standard terminology and notations of the theory of operators in Banach spaces (see, e.g., [6]). All definition, results and details, concerning the notions of frames in Hilbert and Banach spaces, can be found in [1], [2], [5]. Recall only a definition of a Schauder frame for a Banach space (cf. [2] or [5]): a system $\left(x_{k}^{\prime}\right)_{k=1}^{\infty} \subset$ $X^{*},\left(x_{k}\right)_{k=1}^{\infty} \subset X$ is said to be a Schauder frame for $X$, if for every $x \in X$ the series $\sum_{k=1}^{\infty}\left\langle x_{k}^{\prime}, x\right\rangle x_{k}$ converges in $X$ to $x$. An evident generalization is given by the following

Definition 1.1. Let $T \in L(X, W),\left(x_{k}^{\prime}\right)_{k=1}^{\infty} \subset X^{*},\left(w_{k}\right)_{k=1}^{\infty} \subset W$. We say that $\mathcal{F}:=$ $\left(\left(x_{k}^{\prime}\right)_{k=1}^{\infty},\left(w_{k}\right)_{k=1}^{\infty}\right)$ is an O-frame (operator frame) for $T$, if for every $x \in X$ the series

2010 Mathematics Subject Classification. 46B28.

Key words and phrases. Approximation of operators, bounded approximation property, frames. Communicated by. S.K. Kaushik 